\title{
Reoperação nas esotropias congênita e essencial adquirida não acomodativa
}

\author{
Reoperation in congenital and essential acquired non acomodative esotropia \\ Fábio Ejzenbaum ${ }^{1}$, Adele Christina Manso Marques² ${ }^{2}$ Janine Radd Ferreira Pinto ${ }^{3}$, Carlos Ramos Souza-Dias ${ }^{3}$, Mauro Goldchmit ${ }^{2}$
}

\section{RESUMO}

Objetivo: Analisar os resultados das reoperações nas esotropias congênita e essencial adquirida não acomodativa.

Métodos: Foram avaliados retrospectivamente 393 prontuários de pacientes com diagnóstico de esotropia (91 esotropias congênitas e 302 adquiridas) no Departamento de Oftalmologia da Santa Casa de São Paulo, operados entre os anos de 2000 e 2004.

Resultados: No grupo dos portadores de esotropia congênita, 9 pacientes foram reoperados (9,9\%). As indicações para a nova intervenção foram: subcorreções $(3,3 \%)$ supercorreçōes $(2,2 \%)$, anisotropia (V) $(1,1 \%)$, hipotropia $(1,1 \%)$ e divergências visuais dissociadas (2,2\%). No grupo dos portadores de esotropia essencial adquirida não acomodativa 31 pacientes foram reoperados (10,3\%). As indicações para a nova intervenção foram: subcorreções $(n=6,6 \%)$, supercorreções $(n=2 \%)$ e hipertropias $(n=1,7 \%)$.

Conclusões: A porcentagem de reoperação nos casos de esotropia congênita e essencial adquirida não acomodativa foram 9,9\% e 10,2\% respectivamente, com predominância de subcorreções nas indicações para a realização de nova cirurgia. A presença de ambliopia e desvios maiores que $50^{\Delta}$ na esotropia essencial adquirida não acomodativa (EEANA) foram os mais importantes fatores para maus resultados.

Descritores: Esotropia/diagnóstico; Esotropia/cirurgia; Músculos oculomotores/cirurgia; Procedimentos cirúrgicos oftalmológicos; Visão binocular; Reoperação

\begin{abstract}
Purpose: To analyze the results in patients reoperated from congenital and essential esotropia.

Methods: A retrospective chart review of 393 patients who underwent surgery from 2000-2004 was performed. Subjects were divided into two groups: Congenital esotropia (91patients) and essential esotropia (302 cases).

Results: Among congenital cases we had 9 reoperations (9.9\%). There were undercorrections (3.3\%), overcorrections (2.2\%), anisotropia (V) (1.1\%), hypotropia (1.1\%) and dissociatd vertical divergences (2.2\%). Among the essential cases, there were 31 (10.3\%) reoperations due to undercorrections $(n=6.6 \%)$, overcorrections $(n=2 \%)$ and hypotropias (1.7\%).

Conclusions: Outcomes reoperations rates were $9.9 \%$ and $10.2 \%$ between congenital and essential esotropias with a higher rate of undercorrections. Amblyopia in both groups and deviations higher than $5^{4}$ in essential esotropias seems to be the most important factors for poor results.
\end{abstract}

Keywords: Esotropia/diagnosis; Esotropia/surgery; Oculomotor muscles/surgery; Ophthalmologic surgical procedures; Vision, binocular; Reoperation

\section{INTRODUÇÃO}

As reoperações são muito comuns nas esotropias (ET) congênita (EC) e essencial adquirida não acomodativa (EEANA) com incidências muito variáveis entre os estudos.

Aparentemente, as taxas de reintervenção cirúrgica são mais altas na EC em comparação com a EEANA, pois como os pacientes são operados em média até os 18 meses de vida existem limitações do exame pré-operatório, bem como podem ocorrer o aparecimento tardio de complicações como o desvio vertical dissociado (DVD), hiperfunção de músculos oblíquos e desvios verticais ${ }^{(1)}$. Dentre os fatores que influenciam no resultado cirúrgico, a magnitude do ângulo de desvio pré-operatório, e a precocidade da cirurgia parecem ser os fatores mais importantes nestes pacientes ${ }^{(2)}$.

A ET residual é o desvio mais comum nas indicações de reoperações, ela ocorre de acordo com a literatura, entre 20\% e 40\% dos casos, independentemente do ângulo de desvio pré-operatório e do planejamento cirúrgico(3). Os fatores associados mais comuns se devem à falta de colaboração do paciente à semiologia, ao planejamento inadequado, à acomodação residual e à presença de ambliopia ${ }^{(4)}$.

A exotropia consecutiva é observada em 10\% a 27\% dos casos, principalmente na $\mathrm{EC}^{(5-7)}$. Dentre as principais causas relatadas, estão a ambliopia, a limitação pós-operatória de adução após grandes retrocessos do reto medial, a ausência de estereopsia e a cirurgia simultânea em 3 ou 4 músculos retos horizontais ${ }^{(5,7,8)}$.

O objetivo deste estudo foi observar a incidência de reoperações, e possíveis fatores associados às reintervenções cirúrgicas nos casos de EC e EEANA.

\section{MÉTODOS}

Foram analisados retrospectivamente os prontuários de 393 casos de esotropias operadas no Departamento de Oftalmologia da
Funding: No specific financial support was available for this study.

Disclosure of potential conflicts of interest: F. Ejzenbaum, None; A.C.M. Marques, None; J.F.R. Pinto, None; C.R.Souza-Dias, None; M.Goldchmit, None.

Correspondence address: Dr. Fábio Ejzenbaum. Rua Albuquerque Lins, 1.144 - Apto. 82 - São Paulo SP - 01230-000 - Brazil - Email: fabio_e@terra.com.br

Editorial Note: After completing the confidential analysis of the manuscript, ABO discloses, with her agreement, the name Dr. Patricia Grativol Costa as a reviewer. We thank her effort and expertise in participating in this process. 
Santa Casa de São Paulo, entre os anos de 2000 e 2004. Destas, 91 eram EC e 302 EEANA.

Os casos foram analisados com relação ao resultado pós-operatório, enfocando a presença ou não de ambliopia, o número de reoperações, as medidas dos desvios pré e pós-operatórios, a presença de anisotropias, DVD e hipertropias.

Foram estabelecidos como critérios de exclusão os casos que não tiveram todos os procedimentos cirúrgicos realizados na Santa Casa de SP, pacientes com seguimento pós-operatório inferior a cinco meses, casos com fator acomodativo associado e presença de outras doenças oftalmológicas que pudessem influenciar no resultado cirúrgico.

Todos os pacientes submeteram-se ao exame oftalmológico pré-operatório, que consistiu da medida da acuidade visual (quando possível), refratrometria sob cicloplegia e fundoscopia. O desvio pré-operatório foi medido com o uso de prismas com os métodos de prisma e cover ou Krimsky, para longe e perto. Ambliopia foi definida como diferença de duas linhas na medida da acuidade visual com a melhor correção óptica, ou forte preferência por um dos olhos, sem alternância, em crianças pré-verbais.

Quanto ao planejamento cirúrgico das reoperações, foram levadas em consideração as seguintes variáveis: quantidade de milímetros na primeira cirurgia, número de músculos operados, desvio residual e presença de limitação de adução/abdução. Com base nesses achados foram programadas as reintervenções, tentando preservar o maior número possível de artérias ciliares.

O estudo teve aprovação do Comitê de Ética da Santa Casa de São Paulo (059/09).

\section{RESULTADOS}

No grupo de EC, a taxa de reoperação foi de 9,9\%, com seguimento de 19,3 \pm 15 meses (5 a 48 meses). A idade média da primeira cirurgia foi de $46,5 \pm 27,2$ meses ( 8 a 80 meses). O desvio pré-operatório antes da primeira cirurgia nos pacientes reoperados foi de ET $45,6^{\Delta} \pm 10,5^{\Delta}\left(30^{\Delta}-60^{\Delta}\right)$.

Os nove casos tiveram como indicações para reoperação: subcorreção (3,3\%), supercorreção (2,2\%), DVD tardia (2,2\%), anisotropia em $\vee(1,1 \%)$ e hipotropia $(1,1 \%)$

As características principais dos casos reoperados de EC podem ser observadas no quadro 1.

Entre os 91 casos com EC, 10 eram amblíopes, destes 5 (50\% dos amblíopes) foram reoperados; quanto à disfunção de músculos oblíquos, dentre o total de 32 casos, 4 (12,5\%) tiveram segunda intervenção (1 caso como motivo principal da reoperação, 1 associado à DVD e 2 casos associados a subcorreção); entre os 14 casos de DVD, 2 (14,2\%) foram reoperados.

As comparações entre as frequências de reoperações dos pacientes com desvios pré-operatórios maior ou menor de $50^{\Delta}$, presença de ambliopia, DVD e disfunção de oblíquos pode ser observada no quadro 2.
No grupo de EEANA, a taxa de reoperação foi de 10,3\% com seguimento de $20 \pm 17$ meses ( 5 a 72 meses). A idade média da primeira cirurgia foi de 19,12 $\pm 12,64$ anos (1 a 51anos). O desvio pré-operatório antes da primeira cirurgia nos pacientes reoperados foi de $E T 46,1^{\Delta} \pm 17,2^{\Delta}\left(20^{\Delta}\right.$ a $\left.80^{\Delta}\right)$, sendo que 17 casos tinham desvio menor que $50^{\Delta}$ e 14 tinham desvio maior que $50^{\Delta}$.

Dos 31 casos, as indicações para reoperação foram: subcorreção $(6,6 \%)$, supercorreção (2\%), hipertropia isolada (1,7\%). Três casos tiveram terceira intervenção, uma supercorreção que após a segunda cirurgia ficou subcorrigida, uma anisotropia em A e uma subcorreção que permaneceu com ET.

As características principais dos pacientes reoperados de EEANA podem ser observadas no quadro 3

Entre os 302 casos com EEANA, 101 eram amblíopes, destes 20 (19,8\% dos amblíopes) foram reoperados; quanto à disfunção de músculos oblíquos, 10 dos 94 casos (10,6\%) tiveram segunda intervenção. Quatorze pacientes tinham hipertropias pré-operatórias, destes, 5 casos (36\% das hipertropias) foram reoperados, as características principais dos casos reoperados estão no quadro 4.

As comparações entre as frequências de reoperações dos pacientes com desvios pré-operatórios maior ou menor de $50^{\Delta}$, presença de ambliopia, hipertropias e disfunção de oblíquos pode ser observada no quadro 5.

Quanto à quantidade de re-retrocesso ou re-ressecção necessários para corrigir o desvio, foram selecionados apenas 6 casos que tiveram re-retrocessos puros do músculo reto medial, com variação de 3 a $5 \mathrm{~mm}$ ( $4 \pm 1 \mathrm{~mm}$ ). A quantidade média de correção foi de 12 $\pm 4,2^{\Delta}$, ou seja, $3^{\Delta} / \mathrm{mm}$ de retrocesso.

\section{DISCUSSÃO}

As principais indicações de reintervenção cirúrgica nas EC foram subcorreções, supercorreções e DVD.

Alguns estudos identificaram alguns fatores responsáveis pela reoperação na EC, como ângulo de desvio pré-operatório ${ }^{(9)}$, ambliopia ${ }^{(9)}$, presença de nistagmo ${ }^{(9)}$, disfunção de músculos oblíquos inferiores ${ }^{(10)}$ e DVD ${ }^{(10)}$

Trigler, Siatkowski(2) observaram 149 pacientes com EC. Desses, 34\% (51 casos) precisaram ser reoperados. Algumas características contribuíram para a reintervenção cirúrgica, como a ambliopia, cirurgia realizada antes dos 15 meses de vida e desvio maior que $30^{\Delta}$. Observou-se em nosso estudo, alta frequência de reoperação entre os amblíopes ( $50 \%$ dos casos), inclusive com teste de Fisher significante.

Ainda quanto ao tamanho do desvio pré-operatório, alguns autores ${ }^{(11)}$ observaram que desvios maiores que $50^{\Delta}$ têm tendência a subcorreção. As conclusões desses autores não corroboraram com nossos resultados.

Outros autores ${ }^{(1)}$ acompanharam 10 crianças com ET congênita e cirurgia precoce (entre 83 e 159 dias de vida); quatro tiveram

Quadro 1. Características principais dos pacientes reoperados de esotropia congênita (EC)

\begin{tabular}{|c|c|c|c|c|c|c|c|c|}
\hline ID & Sexo & $\begin{array}{c}\text { Idade } \\
\text { (meses) }\end{array}$ & Ambliopia & $\begin{array}{c}\text { Desvio PPO } \\
\text { pré-operatório }\end{array}$ & $\mathbf{R} \mathbf{H}$ & $\begin{array}{l}\text { Motivo principal } \\
\text { da reoperação }\end{array}$ & $\begin{array}{l}\text { Posição } \\
\text { final }\end{array}$ & $\begin{array}{l}\text { Seguimento } \\
\text { (meses) }\end{array}$ \\
\hline 1 & $\mathrm{~F}$ & 40 & $\mathrm{~S}$ & ET $60^{\Delta}+$ DVD & 4 & DVD & ET $10^{\Delta}$ & 6 \\
\hline 2 & $M$ & 8 & $\mathrm{~S}$ & ET $40^{\Delta}$ & 2 & Anisotropia em $\mathrm{V}$ & ET $15^{\Delta}$ & 5 \\
\hline 3 & $M$ & 24 & $\mathrm{~S}$ & ET $40^{\Delta}+20^{\Delta}$ & 2 & Subcorreção & $\mathrm{ET} 2^{\Delta}$ & 16 \\
\hline 4 & $\mathrm{~F}$ & 70 & $\mathrm{~N}$ & ET $40^{\Delta}+40^{\Delta}$ & 2 & DVD & $\mathrm{ET} 5^{\Delta}$ & 6 \\
\hline 5 & M & 80 & $S$ & ET $60^{\Delta}$ & 2 & Subcorreção & ET $12^{\Delta}$ & 12 \\
\hline 6 & $\mathrm{~F}$ & 80 & $\mathrm{~N}$ & ET $50^{\Delta}$ & 2 & Subcorreção & ET $8^{\Delta}$ & 9 \\
\hline 7 & $M$ & 40 & $\mathrm{~S}$ & ET $45^{\Delta}+20^{\Delta}$ & 3 & Hipertropia & $\mathrm{ET} 5^{\Delta}$ & 48 \\
\hline 8 & $\mathrm{~F}$ & 17 & $\mathrm{~N}$ & ET $30^{\Delta}$ & 2 & Supercorreção & ET $4^{\Delta}$ & 36 \\
\hline 9 & $M$ & 60 & $\mathrm{~N}$ & ET $70^{\Delta}$ & 3 & Supercorreção & ET $4^{\Delta}$ & 24 \\
\hline
\end{tabular}

$\mathrm{ID}=$ identificação; $\mathrm{F}=$ feminino; $\mathrm{M}=$ masculino; $\mathrm{S}=\operatorname{sim} ; \mathrm{N}=$ não; $\mathrm{ET}=$ esotropia; $\mathrm{DVD}=$ desvio vertical dissociado; $\mathrm{PPO}=$ posição primária do olhar; $\mathrm{RH}=$ número de retos horizontais operados na $1^{\mathfrak{q}}$ cirurgia; posição final= desvio após reoperação; + = direito sobre esquerdo; - = esquerdo sobre direito 
Quadro 2. Teste de Fisher comparando as frequências entre os amblíopes, pacientes com desvio vertical dissociada (DVD), reoperações de músculos oblíquos e desvios maiores ou menores que $50^{\Delta}$ dos pacientes com esotropia congênita (EC)

\begin{tabular}{lccc}
\hline Variável & Pacientes reoperados & Pacientes não reoperados & $\mathbf{P}$ \\
\hline Amblíopes & 5 & 5 & $\mathbf{0 , 0 0 1}$ \\
Não amblíopes & 4 & 7 & $\mathbf{0 , 6 2 4}$ \\
DVD & 2 & 12 & $\mathbf{0}, 715$ \\
Sem DVD & 7 & 70 & \\
Hiperfunção de oblíquos & 4 & 28 & $\mathbf{0 , 2 8 2}$ \\
Sem hiperfunção de oblíquos & 5 & 54 & \\
Desvio $<50^{\Delta}$ & 5 & 22 & 60 \\
Desvio $>50^{\Delta}$ & 4 & 60 & \\
\hline
\end{tabular}

Quadro 3. Características principais dos pacientes reoperados de esotropia essencial adquirida não acomodativa (EEANA)

\begin{tabular}{|c|c|c|c|c|c|c|c|c|c|}
\hline ID & Sexo & $\begin{array}{l}\text { Idade } \\
\text { (anos) }\end{array}$ & Ambliopia & $\begin{array}{l}\text { Desvio em PPO } \\
\text { pré-operatório }\end{array}$ & $\mathbf{R H}$ & $\begin{array}{l}\text { Motivo principal } \\
\text { da reoperação }\end{array}$ & $\begin{array}{l}\text { Motivo segunda } \\
\text { reoperação }\end{array}$ & $\begin{array}{l}\text { Posição } \\
\text { final }\end{array}$ & $\begin{array}{c}\text { Seguimento } \\
\text { (meses) }\end{array}$ \\
\hline 1 & $\mathrm{~F}$ & 31 & $\mathrm{~s}$ & ET $45^{4}$ & 2 & Subcorreção & & ET $15^{\Delta}$ & 31 \\
\hline 2 & $\mathrm{~F}$ & 31 & S & ET $55^{\Delta}$ & 3 & Subcorreção & & ET $15^{\Delta}$ & 6 \\
\hline 3 & M & 13 & $\mathrm{~N}$ & ET $52^{\Delta}$ & 3 & Subcorreção & & ET $10^{\Delta}$ & 5 \\
\hline 4 & $F$ & 10 & $\mathrm{~N}$ & ET $30^{4}$ & 2 & Subcorreção & & 0 & 7 \\
\hline 5 & $\mathrm{~F}$ & 32 & S & ET $75^{\Delta}$ & 3 & Subcorreção & & 0 & 8 \\
\hline 6 & $\mathrm{~F}$ & 18 & S & ET $45^{\Delta}+15^{\Delta}$ & 2 & Subcorreção & & ET $12^{\Delta}$ & 48 \\
\hline 7 & M & 25 & S & ET $95^{\Delta}$ & 4 & Subcorreção & & 0 & 7 \\
\hline 8 & $\mathrm{~F}$ & 19 & S & ET $50^{\Delta}$ & 3 & Subcorreção & & 0 & 6 \\
\hline 9 & $\mathrm{~F}$ & 14 & S & ET $50^{\Delta}$ & 2 & Subcorreção & & $\mathrm{ET} 5^{\Delta}$ & 8 \\
\hline 10 & M & 8 & s & ET $80^{4}$ & 3 & Subcorreção & & 0 & 10 \\
\hline 11 & $\mathrm{~F}$ & 32 & S & ET $40^{\Delta}$ & 2 & Subcorreção & & ET $8^{\Delta}$ & 36 \\
\hline 12 & $\mathrm{~F}$ & 20 & S & ET $40^{\Delta}+8^{\Delta}$ & 2 & $\mathrm{HT}^{3}$ & & 0 & 36 \\
\hline 13 & $\mathrm{~F}$ & 1 & s & ET $45^{\Delta}+15^{\Delta}$ & 2 & Subcorreção & & 0 & 14 \\
\hline 14 & M & 20 & $\mathrm{~N}$ & ET $50^{\Delta}$ & 3 & Subcorreção & Subcorreção & 0 & 9 \\
\hline 15 & $\mathrm{~F}$ & 6 & $\mathrm{~N}$ & ET $50^{\Delta}$ & 2 & HT & & ET $15^{\Delta}$ & 6 \\
\hline 16 & $\mathrm{~F}$ & 2 & $\mathrm{~N}$ & ET $35^{\Delta}+10^{\Delta}$ & 2 & HT & & 0 & 5 \\
\hline 17 & M & 18 & $\mathrm{~N}$ & ET $20^{\Delta}+25^{\Delta}$ & 1 & Subcorreção & & ET $10^{\Delta}$ & 6 \\
\hline 18 & M & 26 & S & ET $30^{\Delta}+8^{\Delta}$ & 2 & Subcorreção & & 0 & 6 \\
\hline 19 & $\mathrm{~F}$ & 13 & $\mathrm{~N}$ & ET $50^{\Delta}$ & 2 & $\mathrm{HT}$ & & ET $15^{\Delta}$ & 6 \\
\hline 20 & M & 12 & s & ET $20^{\Delta}$ & 2 & Subcorreção & & 0 & 6 \\
\hline 21 & $\mathrm{~F}$ & 41 & $\mathrm{~N}$ & ET $35^{\Delta}+15^{\Delta}$ & 2 & $\mathrm{HT}^{3}$ & & 0 & 5 \\
\hline 22 & M & 51 & S & ET $45^{\Delta}$ & 2 & Subcorreção & & ET $15^{\Delta}$ & 72 \\
\hline 23 & $\mathrm{~F}$ & 32 & s & ET $45^{\Delta}$ & 2 & Subcorreção & & 0 & 5 \\
\hline 24 & $\mathrm{~F}$ & 26 & S & ET $50^{\Delta}$ & 2 & Subcorreção & & 0 & 7 \\
\hline 25 & M & 16 & S & ET $40^{\Delta}$ & 2 & Subcorreção & & ET $12^{\Delta}$ & 13 \\
\hline 26 & $\mathrm{~F}$ & 43 & $\mathrm{~N}$ & ET $30^{4}$ & 2 & Supercorreção & & ET $8^{\Delta}$ & 15 \\
\hline 27 & M & 23 & s & ET $70^{\Delta}$ & 2 & Supercorreção & & 0 & 5 \\
\hline 28 & M & 16 & S & ET $25^{\Delta}$ & 2 & Supercorreção & Subcorreção & ET $15^{\Delta}$ & 15 \\
\hline 29 & $\mathrm{~F}$ & 1 & s & ET $30^{\Delta}$ & 2 & Supercorreção & & ET $6^{\Delta}$ & 6 \\
\hline 30 & $\mathrm{~F}$ & 6 & $\mathrm{~N}$ & ET $50^{\Delta}$ & 2 & Supercorreção & & ET $4^{\Delta}$ & 58 \\
\hline 31 & M & 7 & $\mathrm{~N}$ & ET $40^{4}$ & 3 & Supercorreção & & ET $6^{\Delta}$ & 15 \\
\hline
\end{tabular}

$\mathrm{ID}=$ identificação; $\mathrm{F}=$ feminino; $\mathrm{M}=$ masculino; $\mathrm{S}=$ sim; $\mathrm{N}=$ não; $\mathrm{ET}=$ esotropia; $\mathrm{PPO}=$ posição primária do olhar; $\mathrm{RH}=$ número de retos horizontais operados na ${ }^{\circledR}$ cirurgia; $\mathrm{posição} \mathrm{final=}$ desvio após reoperação; += direito sobre esquerdo; - = esquerdo sobre direito

segunda intervenção e três foram submetidas a duas reoperações. Prieto-Díaz ${ }^{(10)}$ estudou 60 crianças operadas de ET congênita e observou indicação de reoperação em 21 delas: nove por recidiva da esotropia, 10 por exotropia consecutiva e duas por apresentarem DVD descompensada. Nesse mesmo estudo o autor observou que a cirurgia até os 18 meses de vida permitia algum grau de visão binocular. Alguns autores acreditam que a estereopsia pode ser atingida com cirurgia precoce ${ }^{(12,13)}$, contribuindo para um melhor alinhamento pós-operatório.

É importante ressaltar que a média da idade dos pacientes na primeira cirurgia em nosso estudo foi bem maior que outros centros, provavelmente porque os pacientes demoram a chegar ao nosso serviço, devido ao baixo poder econômico. Esse fato cria viés quanto ao aparecimento tardio de desvios associados à EC. Provavelmente devido a essa alta média, nossa taxa de reoperação seja baixa comparada a outros trabalhos.

A esotropia residual é o tipo de desvio pós-operatório mais comum nas reoperações de EC, alguns autores observaram até 40\% de reincidência da $\mathrm{ET}^{(14)}$.

Simonsz et al., e Prieto-Díaz observaram que a DVD representa achado importante na reintervenção tardia da EC. Porém em nosso estudo foi observado que a DVD não foi fator preponderante entre nossas taxas de reoperação.

Dentre os casos de EEANA, houve predominância de subcorreções, supercorreções e hipertropias tardias ${ }^{(10,15)}$.

Características como presença de ambliopia, anisotropia em " $A$ " ou "V" e cirurgia em 3 ou 4 músculos retos parecem ser fatores que contribuem para a necessidade de reoperação ${ }^{(5,16,17)}$. 
Quadro 4. Características principais dos pacientes com esotropia essencial adquirida não acomodativa (EEANA) reoperados de hipertropia

\begin{tabular}{ccccc}
\hline ID & $\begin{array}{c}\text { Desvio em PPo } \\
\text { pré-operatório }\end{array}$ & $\begin{array}{c}\text { Desvio PPo } \\
\text { pós 1 } \mathbf{1}^{\mathbf{a}} \text { cirurgia }\end{array}$ & $\begin{array}{c}\text { Causa da hipertropia } \\
\text { pós-operatória }\end{array}$ & $\begin{array}{c}\text { Posição } \\
\text { final }\end{array}$ \\
\hline 12 & ET $40^{\Delta}+8^{\Delta}$ & ET $20^{\Delta}+20^{\Delta}$ & Hiperfunção Ol direito pós retrocesso & 0 \\
15 & ET $50^{\Delta}$ & ET $15^{\Delta}+20^{\Delta}$ & Hiperfunção Ol direito tardia & ET $15^{\Delta}$ \\
16 & ET $35^{\Delta}+10^{\Delta}$ & Hiperfunção OS esquerdo (pós Berke) & 0 \\
19 & ET $50^{\Delta}$ & ET $15^{\Delta}-12^{\Delta}$ & DVD & ET $15^{\Delta}$ \\
21 & ET $35^{\Delta}+15^{\Delta}$ & ET $4^{\Delta}+12^{\Delta}$ & Hiperfunção OS esquerdo tardia & 0 \\
\hline
\end{tabular}

$\mathrm{ID}=$ identificação; $\mathrm{PPO}=$ posição primária do olhar; + = direito sobre esquerdo; - = esquerdo sobre direito

Quadro 5. Teste de Fisher comparando as frequências entre os amblíopes, pacientes com hipertropias, reoperações de músculos oblíquos e desvios maiores ou menores que $50^{\Delta}$ no grupo esotropia essencial adquirida não acomodativa (EEANA)

\begin{tabular}{lccc}
\hline Variável & Pacientes reoperados & Pacientes não reoperados & P \\
\hline Amblíopes & 20 & 8 & $<0,001$ \\
Não amblíopes & 11 & 190 & $\mathbf{0 , 0 0 1}$ \\
Hipertropia & 5 & 9 & $\mathbf{0}, 601$ \\
Sem hipertropia & 26 & 262 & \\
Hiperfunção de oblíquos & 10 & 84 & $\mathbf{0}, 033$ \\
Sem hiperfunção de oblíquos & 21 & 187 & 201 \\
Desvio $<\mathbf{5 0}^{\Delta}$ & 17 & 70 & \\
Desvio $>\mathbf{5 0}^{\Delta}$ & 14 & & \\
\hline
\end{tabular}

Acreditamos que o grande número de amblíopes reoperados $(19,8 \%)$ reflita o achado primordial para a indicação de reintervenção cirúrgica no nosso estudo. Nas exotropias consecutivas, segundo outros autores, bem como nas subcorreções como observado no estudo de Simonsz et al., o principal fator causal parece ser a ambliopia ${ }^{(16-18)}$

A magnitude do ângulo inicial de desvio também foi fator que pode ter contribuído para o mau resultado em alguns casos de EEANA. Dos 31 pacientes reoperados, apenas sete tinham desvio menor ou igual a $30^{\Delta}$, sendo que14 tinham desvio maior que $50^{\Delta}$. Obtivemos resultado significante quando comparamos desvios maiores ou menores que $50^{\Delta}$ anteriores a primeira cirurgia.

As hipertropias também representaram importante motivo de reoperações $(36 \%, p=0,001)$. O principal fator causal para o mau resultado nas hipertropias parece ser a intervenção sobre os músculos oblíquos, pois dos cinco casos em que a hipertropia isolada foi a causa de reoperação, apenas um paciente não tinha disfunção de músculos oblíquos.

Assim como nas EC, as subcorreções responderam pelo maior número de reintervenções. Acreditamos que esse fato se deva à tendência de nosso serviço de planejamentos cirúrgicos mais moderados, visando caso haja necessidade de reoperação, agir sobre as subcorreções.

Analisando os seis re-retrocessos de músculos retos mediais, observamos que cada milímetro correspondeu a correção de aproximadamente $3^{\Delta}$. Outros autores observaram em 12 pacientes que foram submetidos a re-retrocessos dos retos mediais, correção de aproximadamente $2,5^{\Delta}$ para cada milímetro de retrocesso ${ }^{(19)}$.

\section{CONCLUSÕES}

A porcentagem de reoperações nos casos de EC e de EEANA foi de $9,9 \%$ e $10,2 \%$ respectivamente.

Houve predominância das subcorreções, e a presença de ambliopia foi a principal causa responsável pelos insucessos cirúrgicos.

Observou-se também que desvios de maior magnitude (maior que $50^{\Delta}$ ) representam fator de risco para reoperações nos casos de EEANA.

\section{REFERÊNCIAS}

1. Helveston EM, Neely DF, Stidham DB, Wallace DK, Plager DA, Sprunger DT. Results of early alignment of congenital esotropia. Ophthalmology. 1999;106(9):1716-26. Comment in: Ophthalmology. 2001;108(1):5-7. Ophthalmology. 2001;108(1):7.

2. Trigler $\mathrm{L}$, Siatkowski RM. Factors associated with horizontal reoperation in infantile esotropia. JAAPOS. 2002:6(1):15-20.

3. Vroman DT, Hutchinson AK, Saunders RA, Wilson ME. Two muscle surgery for congenital esotropia: rate of reoperation in patients with small versus large angles of deviation. JAAPOS. 2000:4(5):267-70.

4. Felius J, Stager DR Jr, Beauchamp GR, Stager DR. Re-recession of the medial rectus muscles in patients with recurrent esotropia. JAAPOS. 2001;5(6):381-7.

5. Bradbury JA, Doran RM. Secondary exotropia: a retrospective analysis of matched cases. J Pediatr Ophthalmol Strabismus. 1993;30(3):163-6. Comment in: J Pediatr Ophthalmol Strabismus. 1994;31(4):212-3.

6. Stager DR, Weakley DR Jr, Everette M, Birch EE. Delayed consecutive exotropia following 7milimeter bilateral medial rectus recession for congenital esotropia. J Pediatr Ophthalmol Strabismus. 1994;31(3):147-50.

7. Oguz V, Arvas S, Yolar M, Kizilkaya M, Tolun H. Consecutive exotropia following strabismus surgery. Ophthalmologica. 2002;216(4):246-8.

8. Spaeth EB. Factors related to postoperative exotropia. J Pediatr Ophthalmol 1972;9:47-51

9. Hiles DA, Watson BA, Biglan AW. Characteristics of infantile esotropia following early bimedial rectus recession. Arch Ophthalmol. 1980;98(4):697-703.

10. Prieto-Díaz J. Surgical management of congenital (or infantile esotropia) with Ciancia Syndrome. In: $2^{\text {nd }}$ International Symposium on Strabismus 1988: Barcelona: Jims. p. 303

11. Robb RM, Rodier DW. The variable clinical characteristics and course of early infantile esotropia. J Pediatr Ophthalmol Strabismus. 1987;24(6):276-81.

12. O' Keefe M, Abdulla N, Bowell R, Lanigan B. Binocular function and amblyopia after early surgery in infantile esotropia. Acta Ophthalmol Scand. 1996;74(5):461-2.

13. Wright $K$, Edelman PM, McVey JH, Terry AP, Lin M. High grade stereoacuity after early surgery for congenital esotropia. Arch Ophthalmol. 1994:112(7):913-9.

14. Bietti GB, Bagolini B. Problems related to surgical overcorrection in strabismus surgery J Pediatr Ophthalmol Strabismus. 1965;2:11-4.

15. Simonsz HJ, Kolling GH, Unnebrink K. Final report of the early vs.late infantile strabismus surgery study (ELISSS), a controlled, prospective, multicenter study. Strabismus. 2005; 13(4):169-99. Erratum in: Strabismus. 2006;14(2):127-8.

16. Mittelman D, Folk ER. The surgical treatment of overcorrected esotropia. J Pediatr Ophthalmol Strabismus. 1979:16(3):156-9.

17. Patel AS, Simon JW, Lininger LL. Bilateral lateral rectus recession for consecutive exotropia. JAAPOS. 2004:4(5):291-4

18. Donaldson M J, Forrest MP, Gole G A. The surgical management of consecutive exotropia J AAPOS. 2004;8(3):230-6. Comment in: JAAPOS. 2006;10(3):287; author reply 287-8. JAAPOS. 2006;10(3):287.

19. Zhale Rajavi Z, Ghadim H, Ramezani A, Mansoor A, Daneshvar F. Lateral rectus resection versus medial rectus re-recession for residual esotropia: early results of a randomized clinical trial. Clin Experiment Ophthalmol. 2007;35(6):520-6. Comment in: Clin Experiment Ophthalmol. 2008:36(5):489-90; author reply 490-1. 